\title{
Sulla dispersione anomala delle onde magneto-idrodinamiche
}

\author{
P. E. VALLE
}

\section{INTRODUZIONE.}

$\mathrm{E}$ noto che le onde magneto-idrodinamiche hanno una notevole importanza in questioni di fisica cosmica in generale e di geofisica in particolare.

La possibile esistenza di tali onde fu posta in evidenza da $\mathrm{I}$. Alfvén nel 1942. In seguito vari autori hanno condotto ricerche sulla propagazione delle onde magneto-idrodinamiche, anche nel tentativo di rendere meno restrittive le ipotesi fondamentali su cui si basa la teoria di Alfvén.

Non sembra peraltro che sia stata prestata una sufficiente attenzione al fatto che le onde magneto-idrodinamiche presentano il fenomeno della dispersione anomala.

Questa nota ha lo scopo di mettere in evidenza l'andamento di questo fenomeno, il quale potrebbe avere, in qualche caso, un certo interesse.

Nessun cambiamento è stato apportato alle ipotesi fondamentali della teoria di Alfvén. E stata soltanto riveduta la trattazione formale per renderla più semplice e rigorosa.

\section{LE ONDE MAGNETO-IDRODINAMICHE.}

In seno ad un fluido indefinito, omogeneo ed isotropo, esista un campo magnetico primario costante $\mathbf{H}_{0}$. Si supponga che il fluido sia incompressibile, non viscoso, non ferromagnetico e scarico ed abbia una conducibilità elettrica $\sigma$.

Se il fluido viene perturbato, il suo movimento e il campo elettromagnetico, di cui diverrà sede, si condizioneranno reciprocamente.

Ove si prendano in considerazione movimenti sufficientemente lenti da rendere trascurabile la corrente di spostamento nei confronti 
della corrente di conduzione, il sistema delle equazioni del campo elettromagnetico e del moto del fluido, è, con evidente significato dei simboli ed in unità Giorgi,

$$
\begin{aligned}
& \operatorname{rot} \mathbf{H}=\mathbf{J} \\
& \operatorname{rot} \mathbf{E}--\mu \frac{\partial \mathbf{H}}{\partial t} \\
& \mathbf{J}=\sigma(\mathbf{E}+\mu \mathbf{v} \wedge \mathbf{H}) \\
& \varrho \frac{d \mathbf{v}}{d t}=\mu \mathbf{J} \wedge \mathbf{H}-\operatorname{grad}(p+\varrho U)
\end{aligned}
$$

a cui devono essere aggiunte le condizioni

Posto

$$
\begin{aligned}
& \operatorname{div} \mathbf{H}=0 \\
& \operatorname{div} \mathbf{E}=0 \\
& \operatorname{div} \mathbf{J}=0 \\
& \operatorname{div} \mathbf{v}=0
\end{aligned}
$$

$$
\mathbf{H}=\mathbf{H}_{\mathbf{0}}+\mathbf{h}
$$

tenendo conto della identità

$$
\frac{d \mathbf{v}}{d t}=\frac{\partial \mathbf{v}}{\partial t}-\mathbf{v} \wedge \operatorname{rot} \mathbf{v}+\operatorname{grad} \frac{1}{2} v^{2}
$$

e ponendo rot $\mathbf{h}$ al posto di $\mathbf{J}$ nella quarta equazione del sistema [1], si ha

$$
\begin{aligned}
\operatorname{rot} \mathbf{h} & =\mathbf{J} \\
\operatorname{rot} \mathbf{E} & =-\mu \frac{\partial \mathbf{h}}{\partial t} \\
\mathbf{J} & =\sigma\left[\mathbf{E}+\mu \mathbf{v} \wedge\left(\mathbf{H}_{\mathbf{0}}+\mathbf{h}\right)\right] \\
\varrho \frac{\partial \mathbf{v}}{\partial t}-\varrho \mathbf{v} \wedge \operatorname{rot} \mathbf{v} & =-\mu \mathbf{h} \wedge \operatorname{rot} \mathbf{h}-\mu \mathbf{H}_{0} \wedge \operatorname{rot} \mathbf{h}- \\
& -\operatorname{grad}\left(p+\varrho U+\frac{1}{2} v^{2}\right) .
\end{aligned}
$$

Le onde magneto-idrodinamiche di Alfvén si ottengono dalle [1'] imponendo che le forze di massa e di pressione siano equilibrate dalle forze magnetiche, in modo che il moto del fluido sia determinato dal solo campo primario $\mathbf{H}_{0}$.

Si dovrà pertanto porre

$$
\begin{gathered}
\operatorname{grad}\left(p+\varrho U+\frac{1}{2}, \cdots\right)=0 \\
\mathbf{v}-\sqrt{\frac{\mu}{\varrho}} \mathbf{h}
\end{gathered}
$$


ossia

$$
\operatorname{grad}\left(p+\varrho U+\frac{1}{\underline{2}} \mu \hbar^{2}\right)=0 .
$$

Dalla posizione [5] risulta che le linee di forza di $\mathbf{h}$ coincidono con le linee di flusso della velocità del fluido. Il campo magnetico $h$ è, per cosi dire, congelato nel fluido.

Tenendo conto delle [4] e [5] il sistema [1'] diviene

$$
\begin{aligned}
& \operatorname{rot} \mathbf{h}=\mathbf{J} \\
& \operatorname{rot} \mathbf{E}=-\mu \frac{\partial \mathbf{h}}{\partial t} \\
& \mathbf{J}=\sigma\left(\mathbf{E}+\mu \mathbf{v} \wedge \mathbf{H}_{0}\right) \\
& \varrho \frac{\partial \mathbf{v}}{i \tau}=-\mu \mathbf{H}_{0} \wedge \operatorname{rot} \mathbf{h} .
\end{aligned}
$$

Si esegua ora la divergenza di ambo i membri della terza equazione del sistema [1"]. Tenuto conto delle condizioni [2], si ottiene

ossia

$$
\operatorname{div} \mathrm{v} \wedge \mathbf{H}_{\mathbf{0}}=\mathbf{0}
$$

$$
\mathbf{H}_{0} \times \operatorname{rot} \mathbf{v}=0 .
$$

Questa relazione mostra, in virtù della [5], che la densità di corrente $\mathbf{J}$ è ortogonale al campo primario $\mathbf{H}_{\mathbf{0}}$.

Se poi si moltiplicano scalarmente per $\mathbf{H}_{0}$ ambo $\mathrm{i}$ membri della quarta equazione del sistema $\left[1^{\prime \prime}\right]$, risulta

$$
\frac{\partial}{\partial t}\left(\mathbf{v} \times \mathbf{H}_{0}\right)=0
$$

e quindi, supposta nulla la componente stazionaria della velocità in direzione del campo primario, anche $\mathbf{v}$ ed $\mathbf{h}$ sono ortogonali al campo primario $\mathbf{H}_{\mathbf{0}}$.

Per ottenere un'equazione contenente la sola incognita $h$, si esegua la rotazione di ambo i membri della terza equazione del sistema [1"], esprimendo $\mathbf{J}$ ed $\mathbf{E}$ in funzione di $\mathbf{h}$ mediante la prima e la seconda equazione. Si ha

$$
\operatorname{rot} \operatorname{rot} \mathbf{h}=\mu \sigma\left(\operatorname{rot} \mathbf{v} \wedge \mathbf{H}_{0}-\frac{\partial \mathbf{h}}{\partial t}\right) .
$$

Poichè $\operatorname{div} \mathbf{h}=0$, l'identità

$$
\operatorname{rot} \operatorname{rot} \mathbf{h}=-\Delta \mathbf{h}+\operatorname{grad} \operatorname{div} \mathbf{h}
$$

consente di scrivere la [8] nella forma

$$
\frac{1}{\sigma \mu} \Delta \mathbf{h}+\operatorname{rot} \mathbf{v} \wedge \mathbf{H}_{0}=\frac{\partial \mathbf{h}}{o t} .
$$


Per eliminare $\mathbf{v}$, si derivi la precedente parzialmente rispetto al tempo e vi si introduca la quarta equazione del sistema [ $\left.\mathbf{1}^{\prime \prime}\right]$. Risulta

$$
\frac{1}{\sigma \mu}-\frac{\partial}{\partial t} \Delta \mathbf{h}-\operatorname{rot}\left[\left(\frac{\mu}{\varrho} \mathbf{H}_{0} \wedge \operatorname{rot} \mathbf{h}\right) \wedge \mathbf{H}_{1}\right]=\frac{\partial^{2} \mathbf{h}}{\partial t^{2}} .
$$

Dato che

$$
\left(\mathbf{H}_{0} \wedge \operatorname{roth}\right) \wedge \mathbf{H}_{0}=-\mathbf{H}_{0}\left(\mathbf{H}_{0} \times \operatorname{roth}\right)+H_{0}^{2} \operatorname{roth}
$$

tenuto conto della [5] e della [6], la [9] diviene

$$
\frac{1}{\sigma \mu} \frac{\partial}{\partial t} \wedge \mathbf{h}+\frac{\mu}{\varrho} H_{0}^{2} \Delta \mathbf{h}=\frac{\partial^{2} \mathbf{h}}{\partial t^{2}} .
$$

Si deve quindi cercare una soluzione della [10] soddisfacente alle condizioni

$$
\begin{aligned}
& \operatorname{div} \mathbf{h}=\mathbf{0} \\
& \mathbf{H}_{0} \times \operatorname{rot} \mathbf{h}=0 \\
& \mathbf{H}_{0} \times \mathbf{h}=\mathbf{0} .
\end{aligned}
$$

La velocità, la pressione, la densità di corrente e il campo elettrico, saranno date dalle relazioni

$$
\begin{aligned}
& \mathbf{v}=\sqrt{\frac{\mu}{\varrho}} \mathbf{h} \\
& p=p_{\mathbf{0}}-\varrho U-\frac{\dot{r}}{\dot{L}} h^{2} \\
& \mathbf{J}=\operatorname{rot} \mathbf{h} \\
& \mathbf{E}=\frac{1}{\sigma} \operatorname{rot} \mathbf{h}-\mu \sqrt{\frac{\mu}{\rho}} \mathbf{h} \wedge \mathbf{H}_{0} .
\end{aligned}
$$

In vista dello scopo che si propone di raggiungere questa nota, è sufficiente studiare la propagazione di un'onda elementare piana.

Assunto un riferimento cartesiano col campo primario $\mathbf{H}_{0}$ in direzione dell'asse $z$, le [11] si scrivono

$$
\begin{gathered}
\frac{\partial h_{x}}{\partial x}+\frac{\partial h_{v}}{\partial y}=0 \\
\frac{\partial h_{v}}{\partial x}-\frac{\partial h_{x}}{\partial y}=0 \\
h_{z}^{\prime}=0 .
\end{gathered}
$$


Se ci si limita a considerare un'onda polarizzata nel piano $x z$, preso l'asse $y$ coincidente con la direzione $\mathbf{h}$, risulta

$$
h_{x}=0 \text {. }
$$

Le $\left[11^{\prime}\right]$ mostrano poi che $h_{\mathrm{y}}$ non dipende dalle coordinate $x$ ed $y$, pertanto la [10] diviene

$$
\frac{1}{\sigma \mu} \frac{\partial^{3} h_{y}}{\partial t \partial z^{2}}+\stackrel{\mu}{\varrho} \ddot{\sim}_{0}{ }^{2} \frac{\partial^{2} h_{y}}{\partial z^{z}}-\frac{\partial^{2} h_{y}}{\partial t^{z}} .
$$

Un'integrale della precedente equazione ha la forma

$$
h_{y}=h_{0 y} e^{j(\omega t-f z)}
$$

con

$$
f^{2}\left(\frac{\mu}{\varrho} H_{0}^{2}+j \frac{\omega}{\sigma_{\ddot{u}}}\right)=\omega^{2}
$$

Dalle [12] risulta inoltre

$$
\begin{aligned}
v_{y} & =n_{m y} / \frac{\mu}{\varrho} e^{j(\omega t-f z)} \\
p & =p_{0}-\varrho U-\frac{\mu}{2} h_{0 y}^{2} e^{2 j(\omega t-f z)} \\
J_{x} & =j f h_{0 y} e^{j(\omega t-f z)} \\
E_{x} & =\left(\frac{j f}{\sigma}-\mu / \frac{\mu}{\varrho} H_{0}\right) h_{0 y} e^{j(\omega t-f z)} .
\end{aligned}
$$

Le relazioni [13] e [12'] mostrano che le onde magneto-idrodinamiche sono onde trasversali, le quali si propagano in direzione del campo primario $\mathbf{H}_{\mathbf{0}}$.

LA DISPERSIONE ANOMALA DELLE ONDE MAGNETO-IDRODINAMICHE.

La velocità di fase e il coefficiente di assorbimento delle onde magneto-idrodinamiche, si ottengono dalla [14].

Indicando con $\lambda$ la lunghezza d'onda e con $k$ il coefficiente di assorbimento, la [14] diviene

$$
\frac{2 \pi}{\lambda}-j k=\frac{\omega}{\sqrt{\frac{\mu}{\varrho} H_{0}{ }^{2}+j \frac{\omega}{\sigma \mu}}} .
$$


Eे conveniente introdurre la frequenza ridotta

$$
q=\frac{\omega}{\omega_{0}}
$$

dove

$$
\omega_{0}=\frac{\sigma \mu^{2} H_{0}^{2}}{\varrho}
$$

e la velocità delle onde magneto-idrodinamiche nel caso che la conducibilità sia infinita, cioè

$$
V_{0}=H_{0} \sqrt{\frac{\mu}{\varrho}} .
$$

L'equazione [15] risolta rispetto a $2 \pi / \lambda$ ed a $k$ fornisce

$$
\begin{gathered}
\frac{2 \pi}{\lambda}-\frac{\omega_{0} q}{V_{0} \sqrt{2}} \frac{\sqrt{\sqrt{1+q^{2}}+1}}{\sqrt{1+q^{2}}} \\
k=\frac{\omega_{0} q}{V_{0} \sqrt{2}} \frac{\sqrt{\sqrt{1+q^{2}}-1}}{\sqrt{1+q^{2}}} .
\end{gathered}
$$

Se si indica con $V$ la velocità di fase e si pone

$$
k_{0}=-\frac{V_{0}}{\omega_{0}}
$$

i valori ridotti della velocità di fase e del coefficiente di assorbimento risultano

$$
\frac{V_{0}}{V}=\frac{\sqrt{2} \sqrt{1+q^{2}}}{\sqrt{\sqrt{1}+q^{2}}+1}
$$

0 anche

$$
\frac{V}{V_{0}}=\frac{12}{q} \sqrt{1+q^{2}} \sqrt{\sqrt{1+q^{2}}-1}
$$

e

$$
\frac{k}{k_{0}}=\frac{q}{\sqrt{2}} \frac{\sqrt{\sqrt{1+q^{2}}-1}}{\sqrt{1+q^{2}}} .
$$

Si osservi che se $q$ tende a zero, la velocità di fase tende a $V_{0}$ e il coefficiente di assorbimento tende a zero, mentre se $q$ cresce, crescono la velocità di fase e il coefficiente di assorbimento. 
Pertanto, ferme restando le altre grandezze, la velocità di fase delle onde magneto-idrodinamiche cresce con la frequenza.

Come è noto tale fenomeno prende il nome di dispersione anomala.

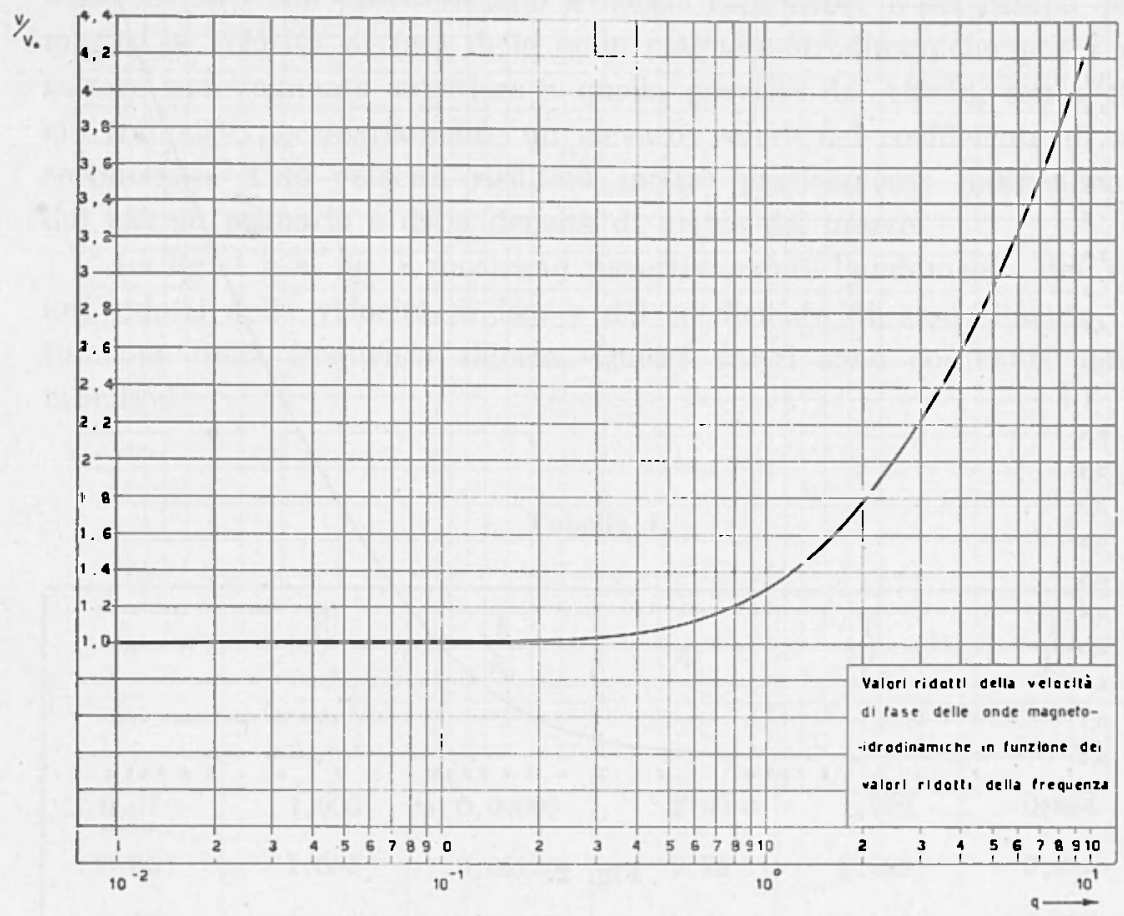

Fig. 1.

Dato che la dispersione non interviene se $q \ll 1$, mentre è sensibile per $q \gg 1$, conviene sviluppare in serie l'espressione della velocità di fase e del coefficiente di assorbimento nei due casi.

a) $q \ll 1$, ossia $\omega \ll \sigma \mu V_{0}^{2}$.

La [22] e la [23] forniscono

$$
\begin{gathered}
V \sim V_{0} \\
k=\frac{1}{2} \cdot \frac{\omega^{2}}{\sigma \mu V_{0}^{3}} .
\end{gathered}
$$


Queste relazioni mostrano che se la conducibilità del mezzo è grande, le onde magneto-idrodinamiche si propagano come previsto da Alfvén.

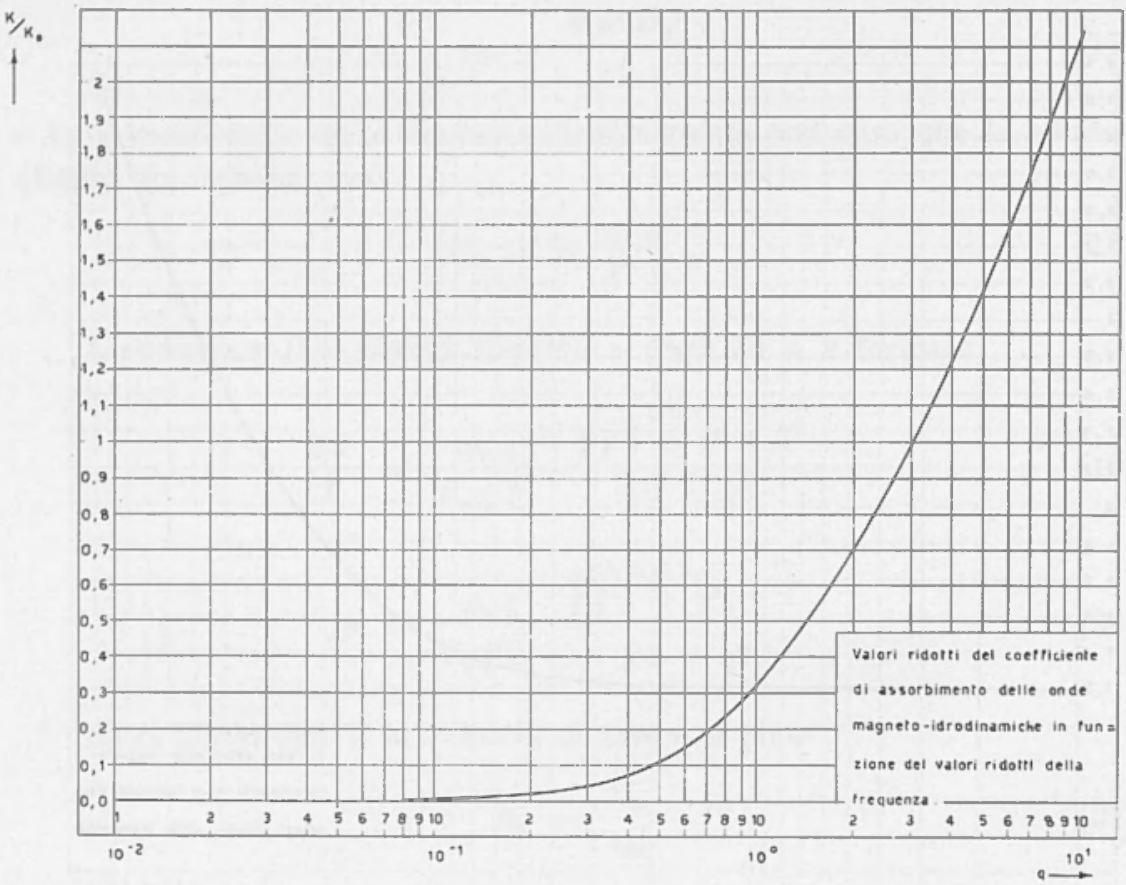

Fig. 2.

b) $q \gg 1$, ossia $\omega \gg \sigma \mu V_{0}^{2}$.

La [22] e la [23], in questo caso, divengono

$$
\begin{aligned}
& V \simeq \sqrt{\frac{2 \omega}{\sigma \mu}}\left(1-\frac{1}{2} \frac{\sigma \mu V_{0}^{2}}{\omega}\right) \\
& k \simeq V_{0}^{2} \sqrt{\frac{\omega \sigma \mu}{2}}\left(1-\frac{1}{2} \frac{\sigma \mu V_{0}^{2}}{\omega}\right) .
\end{aligned}
$$

La prima delle precedenti relazioni mostra che, in questo caso, la velocità di fase tende a divenire indipendente dalla densità di massa del mezzo e del campo primario al crescere della frequenza. 
La possibilità che onde siffatte abbiano un basso coefficiente di assorbimento dipende dalla conducibilltà del mezzo, poichè al diminuire della conducibilltà la velocità di fase cresce e il coefficiente di assorbimento decresce.

Si può quindi concludere che per opportuni valori della frequenza, delle costanti che caratterizzano il mezzo conduttore e del campo primario, la velocità di fase delle onde magneto-idrodinamiche potrà risultare notevolmente superiore a quella prevista da Alfvén, senza che ciò comporti necessariamente un elevato valore del coefficiente di assorbimento. Tale velocità risulterà inoltre praticamente indipendente dal campo primario e dalla densità di massa del mezzo.

La fig. 1 e la fig. 2 mostrano rispettivamente l'andamento dei valori ridotti della velocità di fase e del coefficiente di assorbimento, in funzione della frequenza ridotta. Questi valori sono contenuti nella tabella 1.

Tabella 1.

\begin{tabular}{|c|c|c|c|c|c|}
\hline$q$ & $\frac{V}{\nabla_{0}}$ & $\frac{k}{k_{0}}$ & $q$ & $\frac{V}{\nabla_{0}}$ & $\frac{k}{k_{5}}$ \\
\hline & & & & & \\
\cline { 1 - 2 } 0,01 & 1,000 & 0,00005 & 2,50 & 1,982 & 0,854 \\
0,05 & 1,001 & 0,00125 & 2,75 & 2,088 & 0,922 \\
0,10 & 1,004 & 0,00497 & 3,00 & 2,192 & 0,986 \\
0,25 & 1,023 & 0,0301 & 3,50 & 2,390 & 1,105 \\
0,50 & 1,086 & 0,109 & 4,00 & 2,576 & 1,212 \\
0,75 & 1,178 & 0,212 & 4,50 & 2,752 & 1,311 \\
1,00 & 1,287 & 0,322 & 5,00 & 2,920 & 1,404 \\
1,25 & 1,404 & 0,428 & 6,00 & 3,232 & 1,572 \\
1,50 & 1,523 & 0,527 & 8,00 & 3,787 & 1,865 \\
1,75 & 1,641 & 0,619 & 10,00 & 4,276 & 2,117 \\
2,00 & 1,758 & 0,703 & 20,00 & 6,21 & 3,08 \\
2,25 & 1,871 & 0,781 & 100,00 & 14,2 & 7,04 \\
\hline & & & & & \\
\hline
\end{tabular}


L'andamento della velocità ridotta in funzione del coefficiente di assorbimento ridotto, è illustrato dalla fig. 3 .

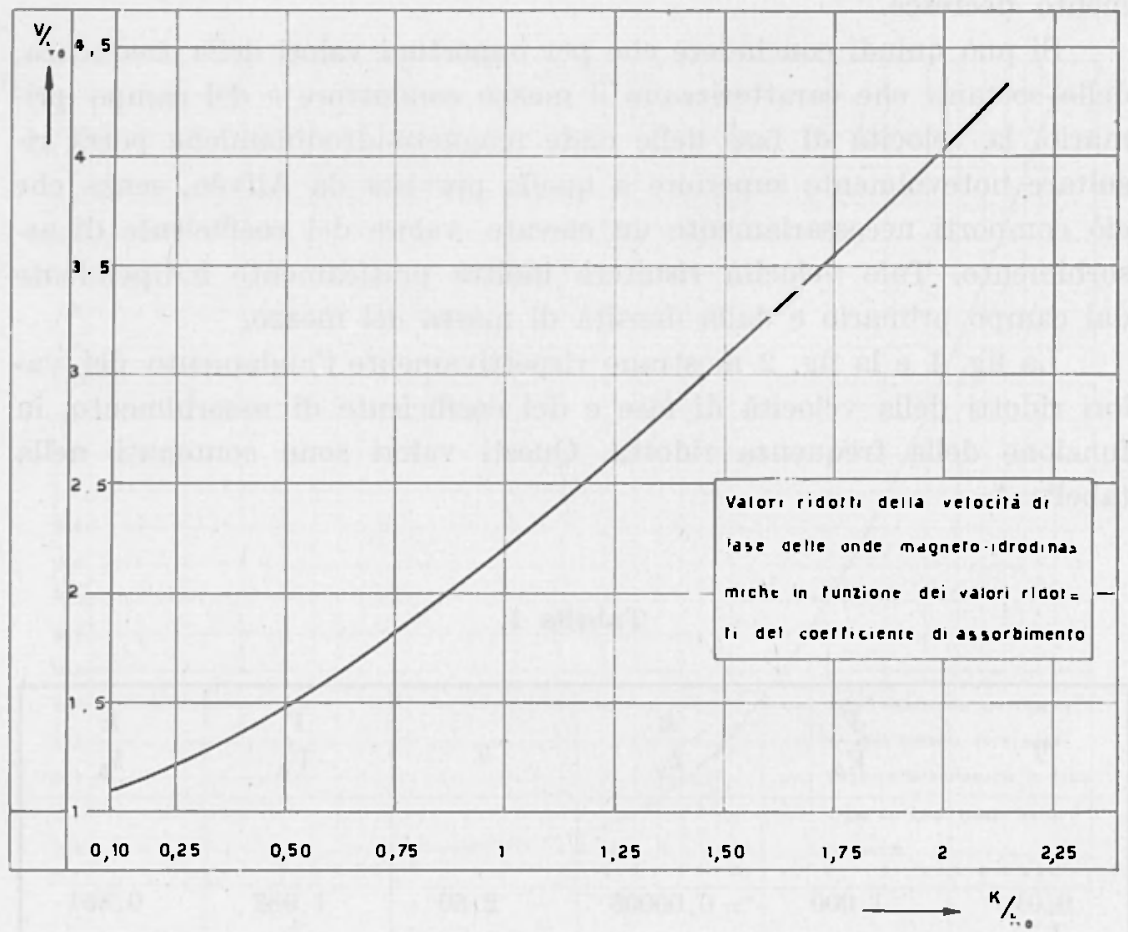

Fig. 3.

Se $q \gg 1$, il coefficiente di assorbimento è approssimativamente proporzionale alla velocità di fase, come risulta dalla [22"'] e dalla [23"].

\section{RIASSUNTO}

Viene valutato l'effetto della dispersione anomala sulla propagazione delle onde magneto-idrodinamiche. Si mostra che, per opportuni valori della frequenza, delle costanti che caratterizzano fisicamente il mezzo conduttore e del campo primario, la velocità di fase delle onde magneto-idrodi- 
SULLA DISPERSIONE ANOMALA DELLE ONDE MAGNETO-IDRODINAMICHE 387

namiche pud̀ risultare notevolmente superiore a quella prevista da Alfvén, senza che ciò comporti necessariamente un elevato valore del coefficiente di assorbimento.

\section{ABSTRACT}

The effect of an anomalous dispersion on the propagation of magnetohydrodynamic waves is discussed in the present article.

It is demonstrated that the phase velocity of the magneto-hydrodynamic waves may be considerably higher than the one envisaged by Alfvèn. 